\title{
A UTILIZAÇÃO DE TERAPIAS ALTERNATIVAS POR PACIENTES EM TRATAMENTO QUIMIOTERÁPICO
}

Camila Bittencourt Jaconodinoํㅜㄹ Simone Coelho Amestoy ${ }^{2}$, Maira Buss Thofehrn ${ }^{3}$

RESUMO: Alguns pacientes oncológicos ao defrontar-se com a doença optam por tratamentos não convencionais a fim de minimizar os sintomas. Este estudo caracterizou-se por uma abordagem quantitativa, exploratória e descritiva e teve como objetivo: conhecer a prevalência da utilização de terapias alternativas por pacientes em tratamento quimioterápico. A amostra foi composta por 65 pacientes, durante suas sessões de quimioterapia, num hospital universitário do interior do Rio Grande do Sul, no mês de setembro de 2006. Após a análise e discussão dos dados evidenciou-se que 69\% dos sujeitos utilizavam práticas alternativas associadas à quimioterapia, sendo a fitoterapia a mais utilizada, com $82 \%$ de adesão. Devido à prevalência elevada de terapias alternativas, acreditamos que os profissionais de enfermagem devem adquirir mais conhecimentos sobre estas práticas, a fim de orientar os pacientes de forma eficaz, melhorando, desta forma, sua qualidade de vida.

PALAVRAS-CHAVE: Terapias Alternativas; Oncologia; Enfermagem.

\section{ALTERNATIVE THERAPIES FOR PATIENTS UNDERGOING CHEMOTHERAPY}

ABSTRACT: Some oncological patients, facing the disease, choose non-conventional therapies in order to minimize the symptoms. Thus, this exploratory-descriptive study with a quantitative approach aimed to: find out about the prevalence of using alternative therapies by patients undergoing chemotherapy. The sample encompassed 65 patients, during chemotherapy sessions, at a university hospital in the interior of Rio Grande do Sul State, in September 2006. After data analysis and discussion, it was evidenced that $69 \%$ of the subjects were using alternative therapies associated to chemotherapy, being phytotherapy the most used one with $82 \%$. Due to the high prevalence of alternative therapies, we believe nursing professionals must obtain further knowledge on these practices, in order to guide patients effectively, thus improving their quality of life.

KEYWORDS: Alternative therapies; Oncology; Nursing.

\section{UTILIZACIÓN DE TERAPIAS ALTERNATIVAS PARA PACIENTES EN EL TRATAMIENTO QUIMIOTERÁPICO}

RESUMEN: Algunos pacientesoncológicos que se deparan con la enfermedad optan por tratamientos no convencionales para reducir al mínimo los síntomas. Así, el objetivo de este estudio fue conocer la prevalencia de la utilización de terapias alternativas para los pacientes en tratamiento de quimioterapia. Su enfoque ES cuantitativo, descriptivo y exploratório. Paticiparon 65 pacientes, mientras sus períodos de sesiones de quimioterapia en un hospital universitario en interior de Rio Grande do Sul, en el mes de septiembre de 2006. Tras el análisis y la discusión de los datos, se destacó el hecho de que 69\% de los enfermos utilizan las prácticas tradicionales asociadas a la quimioterapia, siend la fitoterapia y la más utilizada, con adhesión de 82\% de los miembros. Debido a la alta prevalencia de terapias alternativas, creemos que los profesionales de enfermería deben plantear más conocimientos acerca de esas prácticas, a fin de orientar de manera eficaz a los pacientes, mejorando su cualidad de vida.

PALABRAS CLAVE: Terapias alternativas; Oncología; Enfermería.

${ }^{1}$ Enfermeira. Especialista em Enfermagem com Ênfase em Terapia Intensiva pelo Hospital Moinhos de Vento/ POA.

${ }^{2}$ Enfermeira. Mestranda do Curso de Pós-Graduação da Fundação Universidade do Rio Grande-FURG. Especialista em Enfermagem com Ênfase em Terapia Intensiva pelo POA.

${ }^{3}$ Enfermeira. Doutora em Enfermagem. Professora da Faculdade de Enfermagem e Obstetrícia da Universidade Federal de Pelotas. Coordenadora do Grupo de Pesquisa - NEPEn/UFPel.

Autor correspondente:

Camila Bittencourt Jaconodino

Senador Mendonça 50/202 - 96015-200 - Pelotas-RS

E-mail: cb.jacondino@uol.com.br 


\section{INTRODUÇÃO}

A partir da década de 70 do século passado, a utilização de terapias alternativas se popularizou, tendo sua adesão aumentada para aproximadamente 2 a $3 \%$ ao ano, passando a ser utilizada por vários grupos, entre eles os pacientes oncológicos. A procura por práticas complementares de saúde dá-se principalmente por dois motivos: a insatisfação com a medicina convencional e a busca de afinidades pela utilização de produtos naturais ${ }^{(1)}$. Assim, a população pode optar por tratamentos de menor custo e de fácil aquisição, para poderem solucionar ou amenizar seus problemas de saúde. Estas consistem em técnicas que buscam suprir as reais necessidades dos indivíduos, tanto na prevenção como no tratamento ou cura, considerando-os em todos os seus aspectos, em sua multidimensionalidade ${ }^{(2)}$.

Acreditamos na importância de realizar um trabalho abordando o uso de terapias alternativas a fim de orientar pacientes portadores de neoplasias. Muitas pessoas diante da ansiedade desencadeada pelo diagnóstico dessa patologia saem à procura de tratamentos instantâneos com efeitos extraordinários, objetivando eliminar a doença e minimizar os efeitos associados, porém, acabam, por vezes, colocando suas vidas em risco, submetendo-se à práticas desconhecidas. Neste estudo optamos por abordar algumas terapias alternativas mais difundidas nos meios de comunicação sendo elas: fitoterapia, homeopatia e reiki. A oferta destes tratamentos nos serviços públicos de saúde supõe o reconhecimento oficial de sua utilidade ${ }^{(3)}$.

Durante milhares de anos, os homens vêm utilizando os recursos disponíveis na flora, a fim de promover o tratamento de diversas patologias e este hábito popular denominado de fitoterapia, tem sido propagado de geração a geração no interior dos diversos grupos culturais ${ }^{(4)}$. Em relação ao tratamento do câncer, existem estudos que afirmam a existência de aproximadamente 700 espécies de plantas, as quais apresentam atividades sobre tumores malignos ${ }^{(5)}$. No país, a regulamentação dos medicamentos fitoterápicos vêm sofrendo alterações nos últimos anos. A Agência Nacional de Vigilância Sanitária (ANVISA) tem elaborado normas com este objetivo desde 1995, a mais recente foi a Resolução RDC n.48 de 16 de março de 2004, que dispõe sobre o registro destes medicamentos. Constata-se a preocupação deste órgão público em reconhecer os benefícios e efeitos das plantas medicinais, pois algumas possuem substâncias que podem causar efeitos adversos no organismo humano ${ }^{(4)}$.

Tem-se que a Homeopatia foi introduzida oficialmente no Brasil em 1840, por um ex-comerciante francês e militante socialista denominado Benoit Mure, porém esta só foi reconhecida pelo Conselho Federal de Medicina como uma especialidade médica em 1980. Sua prática no Sistema Único de Saúde ainda é reduzida e existem poucas experiências de serviços de saúde homeopáticos, sendo estes restritos a alguns municípios brasileiros. Está prática baseia-se na cura pelo efeito semelhante, desta forma, utiliza-se uma substância que causa os sintomas de uma doença com 0 intuito de amenizar as dores e desconfortos ${ }^{(6)}$.

A última terapia alternativa que iremos abordar é o reiki, conhecido por ser uma união de energia cósmica universal (REI) que refere-se à dimensão espiritual, com a energia vital individual (KI), a qual circunda nossos corpos mantendo-os vivos. Há registros que comprovam a utilização desta técnica há mais de 8 mil anos, no Tibet. É reconhecida pela Organização Mundial da Saúde e seu uso potencializa o efeito de algumas terapias entre elas a quimioterapia sendo usado como complemento nos tratamentos convencionais maximizando as chances de recuperação ${ }^{(7)}$.

Muitos pacientes oncológicos e suas famílias ao defrontar-se com a gravidade de sua doença buscam suporte físico e emocional de diversas formas, na esperança de cura através do tratamento alopático, porém, este método desencadeia efeitos adversos desagradáveis, como a queda dos cabelos, náuseas e vômitos, entre outros. Isto acaba incentivando a associação de outras medidas terapêuticas a fim de auxiliar à terapêutica convencional, com o objetivo de minimizar os efeitos colaterais.

A partir do exposto este estudo teve como objetivo geral conhecer a prevalência da utilização de terapias alternativas por pacientes em tratamento quimioterápico e como objetivos específicos: investigar o conhecimento dos clientes sobre as terapias alternativas; averiguar a opinião dos pacientes sobre o uso de práticas alternativas, investigar o motivo do uso (ou não) das terapias alternativas e identificar a percepção dos pacientes sobre os possíveis benefícios das terapias alternativas em relação à quimioterapia.

\section{METODOLOGIA}

A pesquisa caracterizou-se por uma abordagem 
quantitativa do tipo exploratória e descritiva, com estudo de delineamento transversal não comparado. Foi desenvolvida em unidade de quimioterapia de um hospital universitário localizado em uma cidade do interior do Rio Grande do Sul, no mês de setembro de 2006. Nesta unidade presta-se assistência ambulatorial à pacientes do Sistema Único de Saúde, com uma média de 300 atendimentos por mês, abrangendo todas as cidades da região sul.

Compuseram o estudo 65 pacientes que submeteram-se à sessões de quimioterapia, sendo escolhidos aleatoriamente e que estavam no mínimo há dois meses realizando este tipo de tratamento, na unidade em questão. O estudo seguiu os procedimentos éticos exigidos pela Resolução nº 196/96 do Conselho Nacional de Saúde do Ministério da Saúde; sendo aprovado pelo Comitê de Ética da instituição. Posteriormente, a coleta dos dados realizou-se uma análise quantitativa a partir das informações obtidas e coletadas nas entrevistas.

\section{RESULTADOS E DISCUSSÃO}

Dos 65 pacientes entrevistados, 57\% pertencem ao sexo feminino, a faixa etária predominante varia em torno dos 55 aos 65 anos, 63\% são católicos, 68\% possuem ensino fundamental incompleto e $29 \%$ trabalham no lar. Em relação ao tempo de submissão a quimioterapia, $42 \%$ iniciaram o tratamento há dois meses.

Quando questionados, 89\% dos participantes afirmaram possuir algum tipo de conhecimento em relação as terapia alternativas, a mais lembrada foi a fitoterapia com 38\%. Grande parte dos entrevistados conhecem algum tipo de terapia alternativa, devido a divulgação dessas práticas em inúmeros veículos de comunicação, proporcionando a todos os tipos de pessoas, em suas mais variadas classes sócioeconômicas a possibilidade de se manterem informadas sobre a existência de diversos tratamentos não convencionais. Além disso, os medicamentos fitoterápicos representam 5\% (280 milhões) do mercado global deste ramo ${ }^{(8)}$.

Ao investigar a opinião sobre a utilização de práticas alternativas $89 \%$ responderam ser favorável e $69 \%$ dos sujeitos da pesquisa fazem uso de algum tipo de terapia complementar. Em relação à utilização das terapias alternativas, cabe destacar que $69 \%$ utilizam tais práticas. Esta adesão pode ser decorrente da busca humana por um maior bem estar e o prolongamento da vida.

As terapias alternativas são uma constante no mundo atual, devido a dificuldade que a medicina tradicional possui de solucionar e tratar alguns agravos à saúde, assim, o ser humano tenta buscar amparo em práticas não tradicionais, mas não menos estudadas ou eficientes como as terapias complementares. Acreditamos que os pacientes oncológicos que participaram deste estudo procuram nos tratamentos alternativos suprir suas necessidades, amenizar suas dores, reduzir os desconfortos, bem como o equilíbrio emocional.

Independente do tratamento escolhido destacamos a importância de oferecer um cuidado terapêutico que consiste em um cuidar diferenciado e profissional baseado na visão singular, solidária e integradora entre o trabalhador de enfermagem e o cliente $^{(9)}$.

As terapias utilizadas pelos sujeitos do estudo foram, a fitoterapia, homeopatia e o reiki, sendo a terapia por ervas e chás medicinais $82 \%$, a mais empregada por estes pacientes (Figura 1).

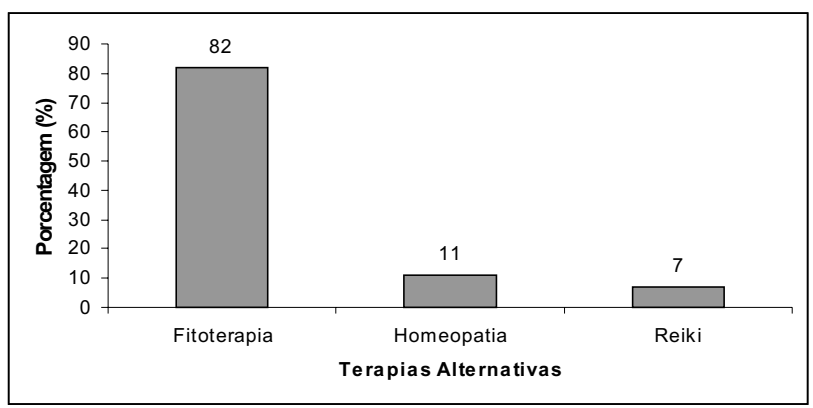

Figura 1 - Terapias alternativas usadas pelos sujeitos do estudo

Pesquisas abordando terapias alternativas correlacionadas aos pacientes portadores de neoplasias evidenciam que a fitoterapia tem sido apontada como a prática de maior adesão. Como mostra um estudo realizado nos países da Europa, no qual investigou-se o modelo de terapia não convencional mais freqüente usado por mulheres com câncer de mama, em que a maioria, $46 \%$, responderam ser adeptas a fitoterapia como tratamento complementar ${ }^{(10)}$.

No Brasil as ervas e chás medicinais também destacam-se por serem as mais citadas por pacientes com câncer como mostra uma pesquisa realizada no Ambulatório da Dor pertencente à Universidade de São Paulo, no qual foram questionados 30 pessoas sobre a utilização de terapias alternativas no controle 
dos sintomas álgicos causados pela doença.

Observamos que grande parte dos sujeitos não dispõe de condição sócio-econômica elevada e a fitoterapia é uma opção que não necessita de custos elevados para o cuidado e aquisição. Os entrevistados que optaram por esse tipo de terapia, como auxiliar ao tratamento quimioterápico, cultivam diversas plantas em seus próprios domicílios e $20 \%$ são agricultores, talvez por este fato evidenciamos o predomínio da fitoterapia.

Ao serem indagados sobre o motivo da utilização de terapias alternativas, $69 \%$ afirmaram que a indicação de familiares e amigos os influenciou na decisão de aliar estas terapias ao tratamento convencional e 31\% relataram a adesão por opção própria.

Alguns entrevistados relataram que passaram a utilizar a fitoterapia e a homeopatia mesmo antes de adquirirem a doença, pois segundo eles, estes métodos não convencionais já eram praticados pelos seus antecedentes e a partir disto este hábito foi passado de geração a geração. O que condiz com alguns trabalhos científicos que comprovaram que a maior parte das informações sobre estas formas de terapias são apresentadas por familiares e amigos ${ }^{(10)}$.

Todas as antigas civilizações têm suas próprias referências históricas relacionadas à fitoterapia, então este método terapêutico, caracteriza-se por estar associado também ao fator cultural e por ser transmitida entre as gerações ${ }^{(11)}$. Cabe esclarecer que a cultura refere-se a valores, normas, crenças e modos de vida aprendidos e compartilhados no interior de um grupo, guiando seu pensar, suas decisões e ações de maneira padronizada.

Entre os entrevistados que não utilizam as terapias alternativas, 35\% responderam ser desnecessária sua utilização por acreditarem nos efeitos potentes da quimioterapia sobre as neoplasias e na amenização dos desconfortos através da administração de medicações que reduzem os efeitos colaterais das drogas antineoplásicas.

Entre os participantes, apenas 25\% desconheciam a existência das terapias alternativas, acreditamos que tal fato decorra da falta de acesso aos meios de divulgação sobre o assunto, 10\% não utilizavam estas terapias por serem contra indicadas pelos seus médicos, está afirmação também foi constatada no estudo de Ribeiro e Campos ${ }^{(12)}$ ao questionarem os sujeitos sobre a não utilização das terapias alternativas. Os autores realizaram um extenso levantamento entre os médicos oncologistas brasileiros com o intuito de entender a relação das terapias alternativas com o tratamento convencional para 0 câncer. Aplicou-se um questionário a 655 membros da Sociedade Brasileira de Câncer, questionando-os a respeito dos tratamentos complementares e sobre a prescrição de tais condutas aos pacientes. Apenas 10\% indicavam as terapias alternativas e, 80,7\% declararam que não as prescreviam devido à falta de comprovação científica de sua eficácia ${ }^{(13)}$.

Percebemos que os pacientes oncológicos tendem a seguir o tratamento de forma criteriosa a fim de proporcionar a cura da doença, portanto acreditamos que por este motivo eles não utilizam outros tratamentos que não são autorizados pelos médicos. Além disso, atualmente, é cada vez mais freqüente a manipulação de medicações e a realização de pesquisas sobre o combate ao câncer, fazendo com que as pessoas façam destas a única forma de tratar a doença, descartando-se a utilização de outras formas terapêuticas até porque as terapias alternativas não costumam ter uma ação rápida como mostra a literatura, ação desejada por muitos pacientes.

Para 44\% dos entrevistados, as terapias alternativas diminuíram os efeitos colaterais da quimioterapia e $40 \%$ afirmaram ter alívio nos sintomas da doença. As terapias alternativas são utilizadas por diversos pacientes com câncer, não apenas como modo de combater os efeitos colaterais do tratamento convencional, mas também por promoverem um aumento da capacidade do organismo de combater a doença $\mathrm{a}^{(12)}$.

Através de nossa experiência profissional identificamos que os pacientes oncológicos são constantemente submetidos a inúmeros procedimentos invasivos, às toxicidades da quimioterapia e, às crises álgicas ocasionadas pela doença. A partir disto, começa uma luta incansável para minimizar ou até mesmo evitar estes acontecimentos, surgindo então, a necessidade de aderir a práticas complementares associadas ao tratamento médico tradicional, a fim de aumentar a qualidade de vida destas pessoas.

Não existem respostas definidas ou garantias para a recuperação do câncer, mas a abordagem holística desperta uma tendência para autocura do paciente, que embora, muitas vezes, não consiga a remissão da doença, promove uma melhor qualidade de vida enquanto aguarda a morte com mais dignidade.

Por volta dos anos cinqüenta do século passado, as terapias alternativas eram relacionadas a crendices 
ou práticas religiosas, porém, cerca de vinte anos após, a utilização de medicamentos homeopáticos, ervas e chás medicinais ganharam maior status e tornaram-se parte de pesquisas científicas as quais tentam comprovar sua eficácia, embora o empirismo que as cercam. Recentemente, o Ministério da Saúde constituiu um grupo de trabalho com a missão de elaborar uma política que permita incorporar ao Sistema Único de Saúde as práticas alternativas, como a fitoterapia e a homeopatia. Perante o uso de plantas medicinais, cabe destacar a existência de um programa denominado Farmácias Vivas, criado há mais de vinte anos, pelo professor Francisco JoséAbreu de Matos da Universidade Federal do Ceará, com o intuito de fornecer medicamentos a população carente desta região ${ }^{(14)}$.

Acreditamos na necessidade de treinamentos para os profissionais da saúde, bem como da enfermagem, para lidar com tais práticas. Assim, poderão fornecer orientações adequadas e essenciais aos pacientes sobre o uso das terapias alternativas a fim de evitar possíveis riscos e agravos à saúde pela utilização inadequada de tais práticas.

Frente a este contexto, o Conselho Federal de Enfermagem (COFEN) regulamentou por meio da Resolução n. 197, de março de 1997, as terapias alternativas como especialidades do enfermeiro ${ }^{(15)}$. Mediante esta determinação do COFEN este profissional obteve o respaldo legal para exercer a função de terapeuta alternativo, mediante a conclusão de cursos de especialização realizados em instituições de ensino reconhecidas e que contenham carga horária mínima de 360 horas $^{(16)}$.

\section{CONSIDERAÇÕES FINAIS}

Atualmente, tem-se observado um aumento na utilização de terapias alternativas. Devido a este motivo resolvemos investigar a prevalência da utilização destas práticas entre os pacientes oncológicos. Os participantes do estudo submeteram-se diariamente a tratamentos com quimioterápicos, os quais produzem efeitos indesejáveis e mutilatórios sejam orgânicos como físicos e neste enfoque muitas pessoas com câncer, procuram diversos tipos de cura, algumas delas não convencionais aliadas a quimioterapia, como mostra esta pesquisa.

O estudo evidenciou que $89 \%$ dos entrevistados conhecem algum tipo de terapia alternativa, sendo a fitoterapia a mais citada 38\%, além disso, 89\% são favoráveis à utilização destas práticas, sendo 69\% adeptos às terapias complementares.

A pesquisa revelou ainda que $82 \%$ dos sujeitos usam ervas e chás medicinais associada ao tratamento convencional, e o motivo principal da utilização é a indicação dos familiares e amigos, 44\% responderam que as terapias alternativas juntamente com a quimioterapia proporciona a diminuição dos efeitos colaterais causados pelas drogas antineoplásicas.

Evidenciou-se que um dos motivos pela preferência da fitoterapia é a fácil aquisição e o baixo custo, além de ser um costume praticado no âmbito familiar sendo indicado por pessoas de seu círculo de amizades, vizinhos e parentes.

Concluímos através deste estudo que grande parte das pessoas aderem às terapias alternativas como cuidado complementar associado ao tratamento para o câncer, pois acima de tudo o tratamento não convencional para o câncer baseia-se em minimizar o sofrimento causado não somente pelos efeitos colaterais, sintomatologia clínica, mas para preencher lacunas que possam originar-se da desestruturação psicológica.

Ainda não foi descoberto algum meio para tratamento de neoplasia em que minimize e evite 0 paciente a submeter-se a efeitos colaterais das drogas antineolásicas e da radiação além da retirada de órgãos os quais possuem valor simbólico, físico e psicológico ao ser humano.

Perante os fatos relatados, almejamos que o estudo em questão sirva como fonte de informação para os profissionais de enfermagem, preparando-os para disponibilizar orientações que possam suprir as dúvidas dos pacientes que utilizam as terapias alternativas.

Por fim, consideramos relevante a aquisição de conhecimentos pelos profissionais da enfermagem e os usuários dos serviços de saúde, sobre diversos tipos de terapias alternativas, bem como seus efeitos colaterais, a fim de minimizarmos os efeitos colaterais do tratamento medicamentoso e obtermos maior qualidade de vida para estes pacientes.

\section{REFERÊNCIAS}

1. Souza M. Terapias alternativas e alternativas perigosas (2004). Disponível em http//:< www. capc.org.br/ terapias.htm>. Acesso em 22 de agosto de 2007.

2. Hill A. Guia das medicinas alternativas: todos os sistemas de cura natural. In: Trovo MM, Silva MJP, Leão ER. Terapias alternativas/complementares no ensino público e privado: análise do conhecimento dos acadêmicos de enfermagem. Rev Latino-Am Enferm. 
2003;11(4):483-9.

3. Palmeira G. A acupuntura no ocidente. Cad Saúde Públ. 1990;6(2):117-28.

4. Turolla MSR, Nascimento ES. Informações toxicológicas de alguns fitoterápicos utilizados no Brasil. Rev Bras Ciênc Farmacol. 2006;42(2):289-306.

5. Flores D. O uso de fitoterapia pelas gestantes: mito ou realidade [monografia]. Pelotas (RS): Universidade Federal de Pelotas; 2003.

6. Monteiro DA, Iriart JAB. Homeopatia no Sistema Único de Saúde: representações dos usuários sobre o tratamento homeopático. Cad Saúde Públ. 2007;23(8):1903-12.

7. Di Carli J. Reiki universal: sistema Usui, Tibetano, Osho e Kahuna. São Paulo: Madras; 2001.

8. Molaniots A, Scott Ja. Complementary and alternative medicine use in beast câncer patientes in Europe. School of Nursing, University os Manchester, Suport Care Cancer; 2005.

9. Amestoy SC, Schwartz E, Thofehrn MB. A humanização do trabalho para os profissionais de enfermagem. Acta Paul Enferm. 2006;19(4):444-9.

10. Marques LC. Fitoterapia. Disponível em http:// $<$ www.connsciência.br >. Acesso em 6 de agosto de 2007.

11. Eldin S, Andrew D. Fitoterapia na atenção primária à saúde. São Paulo: Manole; 2001.

12. Ribeiro $S$ et.al. Use of complementary and alternative medicine by brasilian oncologistis. Europen Journal of Cancer Care. 2005; 14(1):143-8.

13. Planeta Natural. Armas naturais para tratamento do câncer. Disponível em http:// <www.planetanatural.com.br>. Acesso em 2 de agosto de 2007.

14. Silva MIG, Gondim APS, Nunes IFS, Sousa FCF. Utilização de fitoterápicos nas unidades básicas de atenção à saúde da família no município de Maracanaú (CE). Rev Bras Farmacol. 2006;16(4):445-62.

15. Conselho Federal de Enfermagem (BR). Resolução ${ }^{0}$ 197. Estabelece e reconhece as Terapias Alternativas como especialidade e/ou qualificação do profissional de Enfermagem. In: Conselho Regional de Enfermagem. Documentos básicos de enfermagem. São Paulo; 1997.

16. Silva MJP, Benko MA. O uso das terapias alternativas por enfermeiros docentes. Rev Bras Enferm. 1998;5(3):457-68. 